\title{
The Gamow-Teller response within Skyrme random-phase approximation plus particle-vibration coupling
}

\author{
Y. F. Niu ${ }^{1,2}$, G. Colò ${ }^{2} *$ M. Brenna ${ }^{2}$, P.F. Bortignon ${ }^{2}$, and J. Meng ${ }^{1,3,4}$ \\ ${ }^{1}$ State Key Laboratory of Nuclear Physics and Technology, \\ School of Physics, Peking University, Beijing 100871, China \\ ${ }^{2}$ Dipartmento di Fisica, Unversità degli Studi di Milano, \\ and INFN, Sezione di Milano, I-20133 Milano, Italy \\ 3 School of Physics and Nuclear Energy Engineering, \\ Beihang University, Beijing 100191, China and \\ ${ }^{4}$ Department of Physics, University of Stellenbosch, Stellenbosch 7602, South Africa
}

(Dated: July 10, 2018)

\begin{abstract}
Although many random-phase approximation (RPA) calculations of the Gamow-Teller (GT) response exist, this is not the case for calculations going beyond the mean-field approximation. We apply a consistent model that includes the coupling of the GT resonance to low-lying vibrations, to nuclei of the $f p$ shell. Among other motivations, our goal is to see if the particle-vibration coupling can redistribute the low-lying $\mathrm{GT}^{+}$strength that is relevant for electron-capture processes in core-collapse supernova. We conclude that the lowering and fragmentation of that strength are consistent with the experimental findings and validate our model. However, the particle-vibration coupling cannot account for the quenching of the total value of the low-lying strength.

PACS numbers: 21.60.Jz, 23.40.Hc, 24.30.Cz, 25.40.Kv
\end{abstract}

*Electronic address: gianluca.colo@mi.infn.it 


\section{INTRODUCTION}

The Gamow-Teller (GT) resonances are among the clear manifestations of nuclear collective motion. In $(\mathrm{p}, \mathrm{n})$ or $\left({ }^{3} \mathrm{He}, \mathrm{t}\right)$ reactions studied at small angles or zero angular momentum transfer, when the target nucleus is perturbed by an external field proportional to

$$
\hat{O}_{\mathrm{GT}^{-}}=\sum_{i=1}^{A} \vec{\sigma}(i) \cdot \tau_{-}(i),
$$

a broad peak systematically appears in all the nuclei that have been studied in the last three decades [1]. The position and the magnitude of such a peak can be explained only by assuming a coherent effect among particle-hole ( $\mathrm{p}-\mathrm{h})$ excitations in the spin-isospin channel.

Accordingly, much effort has been spent in trying to understand if the systematic study of the GT response can well constrain the nuclear interaction in the spin-isospin channel [27], which will be discussed in this work as well. From the nuclear structure point of view, another interesting phenomenon is the so-called Gamow-Teller quenching. The difference between the total strength associated, respectively, with the $\hat{O}_{\mathrm{GT}^{-}}$and $\hat{O}_{\mathrm{GT}^{+}}$operators [the latter being of course analogous to the former one but proportional to $\tau_{+}(i)$ ], has the model-independent value given by $3(N-Z)$, which is the well-known Ikeda sum rule [8] and will be denoted below by $m_{0}\left(\mathrm{GT}^{-}\right)-m_{0}\left(\mathrm{GT}^{+}\right)$. Only about $60 \%-70 \%$ of this value has been found around the main GT peak [9]. In ${ }^{90} \mathrm{Zr}$, it has been possible to assess the relative importance of different possible quenching mechanisms [10]. In principle, the quenching may be due either to coupling with configurations that are more complicated than the simple p-h ones [11, 12], or to coupling with internal degrees of freedom of the nucleon as with the $\Delta$ particle. Since $93 \%$ of the Ikeda sum rule is found below $50 \mathrm{MeV}$ [10], not much room is left for the latter mechanism.

At the same time the Gamow-Teller and the other spin-isospin charge-exchange nuclear transitions do play a relevant role for particle physics [13, 14] and astrophysics [15]. We will not discuss much their relevance for single and double $\beta$ decay, but we will focus on the connection between GT transitions and the evolution of massive stars at the end of the last hydrostatic burning phase. In fact, if the mass of the iron core exceeds the so-called Chandrasekar mass, then the pressure of the degenerate electron gas is not sufficient to make the system stable against gravitational collapse. In this scenario, one of the main processes that governs the subsequent evolution of the star until an eventual supernova explosion is 
the electron capture both by free protons and by core nuclei in the iron region [15 18].

Extensive tables of electron capture rates, based on realistic estimates of electron capture cross sections, would be needed for supernova simulations. At the electron energies of interest, typically less than $30 \mathrm{MeV}$, electron capture is dominated by the $\mathrm{GT}^{+}$contribution in nuclei around the mass region of Fe [19-22]. When an accurate experimental determination of the corresponding strength is missing, one is obliged to resort to theoretical frameworks such as the shell-model (SM) and the mean-field (MF) or energy-density functional (EDF) based schemes. EDF-based calculations of the electron capture process are worthwhile for several reasons. They are less demanding than SM calculations and are not limited to $s d$ or $f p$ shells. A cross-comparison with the SM can help in reducing the uncertainties associated with the implementation of the EDF-based scheme, and, in a complementary way, the same comparison is certainly instrumental to validate different EDF parameter sets, in the path toward a universal EDF.

In Ref. [21], the electron capture cross sections on several nuclei have been calculated by using a self-consistent implementation of the Skyrme Hartree-Fock plus random-phase approximation (HF plus RPA) model. This model, well known and widely used for many years, has been for the first time extended to finite temperature. The results have been compared with SM results from Refs. [19, 20].

The two models predict cross sections that are not very different at energies around $10-20 \mathrm{MeV}$. At high energies, the SM predictions lie below the HF-RPA ones, whereas at low energies (below about $10 \mathrm{MeV}$ ) the situation is reversed. In fact, the main drawback of HF-RPA seems to be the fact that it predicts a too-high threshold energy for the $\mathrm{GT}^{+}$. Another feature of the finite temperature HF-RPA calculations is a certain spread of the results associated with different parameter sets.

The present work is aimed to increase understanding and try to overcome these open problems. In the simpler zero-temperature case, where one can compare with experimental measurements of the $\mathrm{GT}^{+}$strength, we first study the underlying reasons for the sensitivity of this quantity to the choice of the Skyrme parameter set. Then, we analyze the problem of the threshold energy and the fragmentation of the GT strength. The Skyrme functionals are well known to be unable to describe the single-particle states around the Fermi energy: they tend to predict a too-large single-particle gap, and cannot by definition reproduce the fragmentation of the single-particle strength. As the particle-vibration coupling (PVC) 
approach is one possible improvement in this respect, we will study to which extent a model based on RPA plus particle-vibration coupling can better reproduce the GT strength in the nuclei of interest.

The plan of our paper is therefore the following. We provide first a description of the basic formalism in Sec. [I as well as of the numerical input in Sec. III. The results concerning the sensitivity to the choice of the Skyrme set are discussed in Sec. IV, and the results of RPA plus particle-vibration coupling are discussed in Sec. V. We mainly concentrate on the nucleus ${ }^{60} \mathrm{Ni}$, as a typical system in the mass region of interest. Meanwhile, we also show some results for other nuclei such as ${ }^{56} \mathrm{Ni}$, which has been object of a recent experimental study [23]. We present conclusions in Sec. VI.

\section{SKETCH OF THE THEORETICAL FRAMEWORK}

In the present work, we use the same basic formalism already employed in Ref. [24]. The main difference is that the present PVC calculation is more consistent as we discuss below. The first step is a HF plus RPA calculation of the Gamow-Teller strength. This is done in the same way as in Ref. [5]. The second step consists of implementing the coupling with vibrations.

We start from the solution of the HF equations for a given nucleus $(A, Z)$ using a Skyrme two-body interaction. Then, we set up a discrete basis of both proton-neutron and neutronproton $\mathrm{p}$-h configurations. The continuum is discretized by putting the system in a box and requiring vanishing boundary conditions for the wave functions at the surface of this box. We write the RPA matrix equations and solve them in the model space of the p-h discrete configurations, which is called $Q_{1}$. These equations are well known from textbooks [25] and we shall not discuss them further here. The RPA provides, as a rule, an accurate description of the centroid of giant resonances and the fraction of the energy weighted sum rule exhausted by the mode.

Giant resonances have a quite large damping width. In order to explain it, one needs to go beyond the RPA. As discussed, e.g., in Ref. [24], the width of giant resonances is mainly made up of an escape width $\left(\Gamma^{\uparrow}\right)$ and a spreading width $\left(\Gamma^{\downarrow}\right)$. These are due to different mechanisms, namely to the decay through particle emission and to the coupling with more complicated states of the nuclear spectrum, respectively. The RPA can reproduce 
the escape width if proper coupling to the continuum is implemented, but cannot account for the spreading width.

Here we will follow the formalism of Ref. [24], and only focus on the spreading width which is the leading damping mechanism. The processes in which the energy and angular momentum associated with the vibrational nuclear motion are distributed among more complex internal degrees of freedom do contribute to the spreading width. In order to account for these effects we need a subspace, which we shall call $Q_{2}$ and which is built with a set of "doorway states." We denote these doorway states by $|N\rangle$, and make a physical choice of them in terms of states made up with a p-h excitation coupled to a collective vibration. Note that we use the same Skyrme interaction to calculate both the GT states and all the ingredients to build up the space $Q_{2}$, namely we employ Skyrme-HF single-particle states and vibrations that are calculated consistently in the RPA with the same Skyrme set. In this respect, our scheme is parameter free.

We use the projection formalism to restrict our effective Hamiltonian to the subspace $Q_{1}$ and make the calculations feasible. After truncation of higher-order couplings, the effective Hamiltonian reads

$$
\mathcal{H}(\omega)=Q_{1} H Q_{1}+W^{\downarrow}(\omega)=Q_{1} H Q_{1}+Q_{1} H Q_{2} \frac{1}{\omega-Q_{2} H Q_{2}+i \epsilon} Q_{2} H Q_{1},
$$

where $\omega$ is the excitation energy. This energy-dependent, complex Hamiltonian has complex eigenvalues whose imaginary parts originate from the coupling to the more complicated configurations. We shall denote these complex states as $|\nu\rangle$ in what follows.

In practice, we first diagonalize the RPA Hamiltonian $Q_{1} H Q_{1}$ and obtain the complete basis made up with the RPA states. The creation operators $\mathcal{O}_{\nu}^{\dagger}$ of the states $|\nu\rangle$ can be expressed as a linear combination of the RPA creation operators, namely

$$
\mathcal{O}_{\nu}^{\dagger}=\sum_{\omega_{n}>0} F_{n}^{(\nu)} O_{n}^{\dagger}-\bar{F}_{n}^{(\nu)} \bar{O}_{n}^{\dagger}
$$

where $O_{n}^{\dagger}$ and $\bar{O}_{n}^{\dagger}$ are creation operators of the RPA states $|n\rangle$ lying at positive energy $\omega_{n}$, and of the states $|\bar{n}\rangle$ at the corresponding negative energy $-\omega_{n}$, respectively. Then the eigenvalue equation for the effective Hamiltonian (2), that is,

$$
\left[\mathcal{H}, \mathcal{O}_{\nu}^{\dagger}\right]=\left(\Omega_{\nu}-i \frac{\Gamma_{\nu}}{2}\right) \mathcal{O}_{\nu}^{\dagger}
$$


can be cast in matrix form on the RPA basis as

$$
\left(\begin{array}{cc}
\mathcal{D}+\mathcal{A}_{1}(\omega) & \mathcal{A}_{2}(\omega) \\
-\mathcal{A}_{3}(\omega) & -\mathcal{D}-\mathcal{A}_{4}(\omega)
\end{array}\right)\left(\begin{array}{c}
F^{(\nu)} \\
\bar{F}^{(\nu)}
\end{array}\right)=\left(\Omega_{\nu}-i \frac{\Gamma_{\nu}}{2}\right)\left(\begin{array}{c}
F^{(\nu)} \\
\bar{F}^{(\nu)}
\end{array}\right)
$$

In this latter equation $\mathcal{D}$ is a diagonal matrix with the positive RPA eigenvalues, and the $\mathcal{A}_{i}$ matrices contain the elements associated with the second term of Eq. (2) denoted by $W^{\downarrow}$. The matrix $\left(\begin{array}{cc}\mathcal{D}+\mathcal{A}_{1}(\omega) & \mathcal{A}_{2}(\omega) \\ \mathcal{A}_{3}(\omega) & \mathcal{D}+\mathcal{A}_{4}(\omega)\end{array}\right)$ is complex and symmetric, as it can be seen from their explicit form provided below. The orthogonality and normalization relations of the eigenvectors are

$$
\sum_{n} F_{n}^{(\nu)} F_{n}^{\left(\nu^{\prime}\right)}-\bar{F}_{n}^{(\nu)} \bar{F}_{n}^{\left(\nu^{\prime}\right)}=\delta_{\nu \nu^{\prime}}
$$

Our goal is to extract from the diagonalization of the effective Hamiltonian (2) a physical observable. In particular, we are naturally interested in the response function associated with an external operator such as the GT operator defined in the Introduction. This response function can be written as

$$
R(\omega)=\left\langle 0\left|\hat{O}_{\mathrm{GT}}^{\dagger} \frac{1}{\omega-\mathcal{H}(\omega)+i \eta} \hat{O}_{\mathrm{GT}}\right| 0\right\rangle .
$$

We use a notation that includes both the $\mathrm{GT}^{-}$and $\mathrm{GT}^{+}$cases (one should note that $\hat{O}_{\mathrm{GT}^{-}}^{\dagger}$ is equal to $\hat{O}_{\mathrm{GT}^{+}}$, and vice versa). The strength function is related to Eq. (17) by the well-known relation

$$
S(\omega)=-\frac{1}{\pi} \operatorname{Im} R(\omega)=-\frac{1}{\pi} \operatorname{Im} \sum_{\nu}\left\langle 0\left|\hat{O}_{\mathrm{GT}}\right| \nu\right\rangle^{2} \frac{1}{\omega-\Omega_{\nu}+i \frac{\Gamma_{\nu}}{2}},
$$

where the squared matrix element of the transition operator appears, and not its squared modulus, due to the properties of the eigenvectors $|\nu\rangle$ which form a biorthogonal basis.

We now provide some more details on the calculation of the matrix elements of $W^{\downarrow}$. On the basis of the p-h configurations in $Q_{1}$ space on which the RPA has been solved, the matrix element of $W^{\downarrow}(\omega)$ will be denoted as $W_{p h, p^{\prime} h^{\prime}}^{\downarrow}(\omega)$. If these matrix elements are known, then the matrix elements $\mathcal{A}_{i}$ on the RPA basis are obtained through a straightforward basis transformation,

$$
\begin{aligned}
\left(\mathcal{A}_{1}\right)_{m n} & =\sum_{p h, p^{\prime} h^{\prime}} W_{p h, p^{\prime} h^{\prime}}^{\downarrow}(\omega) X_{p h}^{(m)} X_{p^{\prime} h^{\prime}}^{(n)}+W_{p h, p^{\prime} h^{\prime}}^{\downarrow *}(-\omega) Y_{p h}^{(m)} Y_{p^{\prime} h^{\prime}}^{(n)}, \\
\left(\mathcal{A}_{2}\right)_{m n} & =\sum_{p h, p^{\prime} h^{\prime}} W_{p h, p^{\prime} h^{\prime}}^{\downarrow}(\omega) X_{p h}^{(m)} Y_{p^{\prime} h^{\prime}}^{(n)}+W_{p h, p^{\prime} h^{\prime}}^{\downarrow *}(-\omega) Y_{p h}^{(m)} X_{p^{\prime} h^{\prime}}^{(n)}
\end{aligned}
$$




$$
\begin{aligned}
& \left(\mathcal{A}_{3}\right)_{m n}=\sum_{p h, p^{\prime} h^{\prime}} W_{p h, p^{\prime} h^{\prime}}^{\downarrow}(\omega) Y_{p h}^{(m)} X_{p^{\prime} h^{\prime}}^{(n)}+W_{p h, p^{\prime} h^{\prime}}^{\downarrow *}(-\omega) X_{p h}^{(m)} Y_{p^{\prime} h^{\prime}}^{(n)}, \\
& \left(\mathcal{A}_{4}\right)_{m n}=\sum_{p h, p^{\prime} h^{\prime}} W_{p h, p^{\prime} h^{\prime}}^{\downarrow}(\omega) Y_{p h}^{(m)} Y_{p^{\prime} h^{\prime}}^{(n)}+W_{p h, p^{\prime} h^{\prime}}^{\downarrow *}(-\omega) X_{p h}^{(m)} X_{p^{\prime} h^{\prime}}^{(n)} .
\end{aligned}
$$

$W^{\downarrow}$ is clearly given by

$$
W_{p h, p^{\prime} h^{\prime}}^{\downarrow}(\omega)=\sum_{N} \frac{\langle p h|V| N\rangle\left\langle N|V| p^{\prime} h^{\prime}\right\rangle}{\omega-\omega_{N}} .
$$

The matrix elements at the numerator can be evaluated using Wick's theorem. $W_{p h, p^{\prime} h^{\prime}}^{\downarrow}(\omega)$ turns out to be the sum of the four terms whose diagrammatic representation is shown in Fig. 1 1 and whose analytic expression is

$$
\begin{aligned}
& W^{\downarrow}(1)=\delta_{h h^{\prime}} \delta_{j_{p} j_{p^{\prime}}} \sum_{p^{\prime \prime}, n L} \frac{1}{\omega-\left(\omega_{n}+\epsilon_{p^{\prime \prime}}-\epsilon_{h}\right)+i \eta} \frac{\left\langle p\|V\| p^{\prime \prime}, n L\right\rangle\left\langle p^{\prime}|| V \| p^{\prime \prime}, n L\right\rangle}{\hat{j}_{p}^{2}}, \\
& W^{\downarrow}(2)=\delta_{p p^{\prime}} \delta_{j_{h} j_{h^{\prime}}} \sum_{h^{\prime \prime}, n L} \frac{1}{\omega-\left(\omega_{n}-\epsilon_{h^{\prime \prime}}+\epsilon_{p}\right)+i \eta} \frac{\left\langle h\|V\| h^{\prime \prime}, n L\right\rangle\left\langle h^{\prime}\|V\| h^{\prime \prime}, n L\right\rangle}{\hat{j}_{h}^{2}}, \\
& W^{\downarrow}(3)=\sum_{n L} \frac{(-)^{j_{p}-j_{h^{\prime}}+J+L}}{\omega-\left(\omega_{n}+\epsilon_{p}-\epsilon_{h^{\prime}}\right)+i \eta}\left\{\begin{array}{ccc}
j_{p} & j_{h} & J \\
j_{h^{\prime}} & j_{p^{\prime}} & L
\end{array}\right\}\left\langle p^{\prime}\|V\| p, n L\right\rangle\left\langle h^{\prime}\|V\| h, n L\right\rangle, \\
& W^{\downarrow}(4)=\sum_{n L} \frac{(-)^{j_{p^{\prime}}-j_{h}+J+L}}{\omega-\left(\omega_{n}+\epsilon_{p^{\prime}}-\epsilon_{h}\right)+i \eta}\left\{\begin{array}{ccc}
j_{p} & j_{h} & J \\
j_{h^{\prime}} & j_{p^{\prime}} & L
\end{array}\right\}\left\langle p\|V\| p^{\prime}, n L\right\rangle\left\langle h\|V\| h^{\prime}, n L\right\rangle .
\end{aligned}
$$

In the above formulas, $p$ and $h$ label always particle and hole states, respectively. The corresponding angular momentum and single-particle energy are given by $j_{i}$ and $\epsilon_{i}$. $\hat{j}_{i}^{2}$ is a shorthand notation for $2 j_{i}+1$. The phonon states are labeled by their angular momentum $L$ (only natural parity states are included in our calculations) and by an additional index $n$. The (small) parameter $\eta$ is introduced to mimic couplings beyond the doorway-states approximation: this parameter is set at the value of $500 \mathrm{keV}$.

Up to this point, the scheme is exactly the same as in Ref. [24]. In Ref. [24], the interaction at the particle-vibration coupling vertex $[V$ in Eq. (14)] was approximated by retaining only the $V_{0}$ part in the following momentum-independent terms of the Skyrme p-h force $\left(t_{0}\right.$ and $t_{3}$ terms),

$$
\begin{aligned}
& V_{q q}=V_{0}^{q q} \delta\left(\boldsymbol{r}_{1}-\boldsymbol{r}_{2}\right)+V_{\sigma}^{q q} \delta\left(\boldsymbol{r}_{1}-\boldsymbol{r}_{2}\right) \boldsymbol{\sigma}_{1} \cdot \boldsymbol{\sigma}_{2}, \\
& V_{q \tilde{q}}=V_{0}^{q \tilde{q}} \delta\left(\boldsymbol{r}_{1}-\boldsymbol{r}_{2}\right)+V_{\sigma}^{q \tilde{q}} \delta\left(\boldsymbol{r}_{1}-\boldsymbol{r}_{2}\right) \boldsymbol{\sigma}_{1} \cdot \boldsymbol{\sigma}_{2} .
\end{aligned}
$$




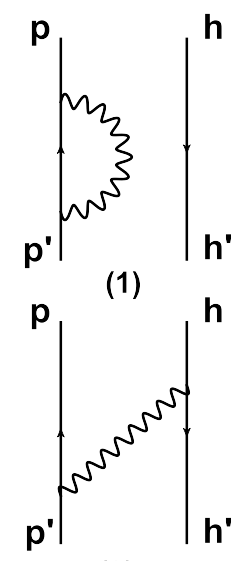

(3)

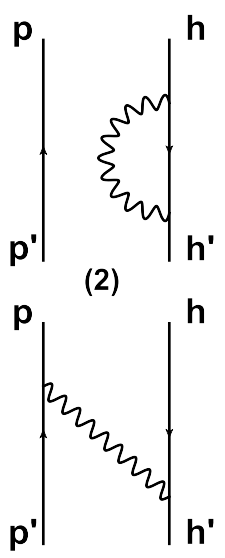

(4)

FIG. 1: Diagrammatic representation of the four terms whose sum gives the matrix element $W_{p h, p^{\prime} h^{\prime}}^{\downarrow}$ The analytic expressions are shown in Eq. (14).

The functions $V_{0}$ and $V_{\sigma}$ depend only on the radial coordinate $r$, and their detailed expressions are

$$
\begin{aligned}
V_{0}^{q q}(r)= & \frac{1}{2} t_{0}\left(1-x_{0}\right)+\frac{1}{16} t_{3}(\alpha+2)(\alpha+1) \rho^{\alpha}(r)-\frac{1}{12} t_{3}\left(x_{3}+\frac{1}{2}\right) \rho^{\alpha}(r) \\
& +\frac{1}{48} t_{3} \alpha(1-\alpha)\left(1+2 x_{3}\right) \rho^{\alpha-2}(r) \rho_{-}^{2}(r)-\frac{1}{12} t_{3}\left(2 x_{3}+1\right) \alpha \rho^{\alpha-1}(r) \rho_{-}(r), \\
V_{0}^{q \tilde{q}}(r)= & \frac{1}{2} t_{0}\left(2+x_{0}\right)+\frac{1}{16} t_{3}(\alpha+2)(\alpha+1) \rho^{\alpha}(r)+\frac{1}{12} t_{3}\left(x_{3}+\frac{1}{2}\right) \rho^{\alpha}(r) \\
& +\frac{1}{48} t_{3} \alpha(1-\alpha)\left(1+2 x_{3}\right) \rho^{\alpha-2}(r) \rho_{-}^{2}(r), \\
V_{\sigma}^{q q}(r)= & \frac{1}{2} t_{0}\left(x_{0}-1\right)-\frac{1}{12} t_{3}\left(1-x_{3}\right) \rho^{\alpha}(r), \\
V_{\sigma}^{q \tilde{q}}(r)= & \frac{1}{2} t_{0} x_{0}+\frac{1}{12} t_{3} x_{3} \rho^{\alpha}(r) .
\end{aligned}
$$

In these formulas, $q$ and $\tilde{q}$ label different charge states of two p-h pairs, where $q \neq \tilde{q}$. $\rho_{-}=\rho_{n}-\rho_{p}$ stands for the density difference of neutron and proton, while $\rho=\rho_{n}+\rho_{p}$ is the total nucleon density. The other symbols are the standard Skyrme parameters. In the present work, we include the whole central Skyrme p-h force, i.e., not only all the $t_{0}$ and $t_{3}$ terms, but also the $t_{1}$ and $t_{2}$ terms with the following form:

$$
\begin{aligned}
V= & \frac{1}{2} t_{1}\left(1+x_{1} P_{\sigma}\right)\left[\boldsymbol{P}^{\prime 2} \delta\left(\boldsymbol{r}_{1}-\boldsymbol{r}_{2}\right)+\delta\left(\boldsymbol{r}_{1}-\boldsymbol{r}_{2}\right) \boldsymbol{P}^{2}\right]\left(1-P_{\sigma} P_{\tau}\right) \\
& +t_{2}\left(1+x_{2} P_{\sigma}\right) \boldsymbol{P}^{\prime} \cdot \delta\left(\boldsymbol{r}_{1}-\boldsymbol{r}_{2}\right) \boldsymbol{P}\left(1+P_{\sigma} P_{\tau}\right)
\end{aligned}
$$

where $\boldsymbol{P}=\frac{1}{2 i}\left(\nabla_{1}-\nabla_{2}\right)$, and $\boldsymbol{P}^{\prime}$ is the Hermitian conjugate of $\boldsymbol{P}$ (acting on the left). $P_{\sigma}=\frac{1}{2}\left(1+\boldsymbol{\sigma}_{1} \cdot \boldsymbol{\sigma}_{2}\right)$ and $P_{\tau}=\frac{1}{2}\left(1+\boldsymbol{\tau}_{1} \cdot \boldsymbol{\tau}_{2}\right)$ are the spin-exchange and isospin-exchange 
operators. This is consistent with the findings of Ref. [26], where it has been shown that the momentum-dependent terms have a non-negligible effect. In Ref. [26] the expression of the reduced matrix elements of $V$ is derived. We report the result here, that is,

$$
\langle i\|V\| j, n L\rangle=\sqrt{2 L+1} \sum_{p h}\left[X_{p h}^{n L} V_{L}(i h j p)+(-)^{L+j_{h}-j_{p}} Y_{p h}^{n L} V_{L}(i p j h)\right]
$$

where the RPA amplitudes appear and $V_{L}$ is the p-h coupled matrix element,

$V_{L}(i h j p)=\sum_{\text {all } m}(-)^{j_{j}-m_{j}+j_{h}-m_{h}}\left\langle j_{i} m_{i} j_{j}-m_{j} \mid L M\right\rangle\left\langle j_{p} m_{p} j_{h}-m_{h} \mid L M\right\rangle\left\langle j_{i} m_{i}, j_{h} m_{h}|V| j_{j} m_{j}, j_{p} m_{p}\right\rangle$.

We briefly recall, for the reader's convenience, the relationship between the present approach in which the full central part of the Skyrme interaction is considered in the PVC vertex, and the approximate one that was used in the past. If, as in Ref. [24], only the $V_{0}(r) \delta\left(\boldsymbol{r}_{1}-\boldsymbol{r}_{2}\right)$ part in $t_{0}$ and $t_{3}$ terms is included in the interaction $V$, the p-h coupled matrix elements have the simple form

$$
\begin{aligned}
V_{L}(i h j p) & =\frac{i^{-l_{i}-l_{h}+l_{j}+l_{p}}}{2 L+1}\left\langle i\left\|Y_{L}\right\| j\right\rangle\left\langle p\left\|Y_{L}\right\| h\right\rangle \int \frac{d r}{r^{2}} V_{0}^{q q^{\prime}}(r) u_{i}(r) u_{j}(r) u_{p}(r) u_{h}(r), \\
V_{L}(i p j h) & =(-)^{L+j_{p}-j_{h}} V_{L}(i h j p),
\end{aligned}
$$

where the radial part of the s.p. (single particle) wave functions, written as $\phi_{n l j m}=$ $\frac{u_{n l j}(r)}{r}\left[Y_{l} \otimes \chi_{1 / 2}\right]_{j m}$, has been introduced. Thus,

$$
\begin{aligned}
\langle i\|V\| j, n L\rangle & =\sqrt{2 L+1} \sum_{p h}\left(X_{p h}^{n L}+Y_{p h}^{n L}\right) V_{L}(i h j p) \\
& =\left\langle i\left\|Y_{L}\right\| j\right\rangle \sum_{q^{\prime}} \int d r V_{0}^{q q^{\prime}}(r) u_{i}(r) u_{j}(r) \delta \rho_{n L}^{\left(q^{\prime}\right)},
\end{aligned}
$$

where $q$ labels the charge of the states $i$ and $j$, and the neutron or proton radial transition density of the state $|n L\rangle$ has been introduced. This is

$$
\delta \rho_{n L}^{(q)}(r)=\frac{1}{\sqrt{2 L+1}} \sum_{p h \in q}\left(X_{p h}^{n L}+Y_{p h}^{n L}\right)\left\langle p\left\|Y_{L}\right\| h\right\rangle \frac{u_{p}(r)}{r} \frac{u_{h}(r)}{r} .
$$

According to the further approximation used in Ref. [24], i.e., namely that a collective state should have mainly isoscalar (or isovector) character so that its isovector (isoscalar) transition density can be neglected, Eq. (21) takes the even simpler form

$$
\langle i\|V\| j, n L\rangle=\left\langle i\left\|Y_{L}\right\| j\right\rangle \int d r V_{0}^{T}(r) u_{i}(r) u_{j}(r) \delta \rho_{n L}^{T}
$$


which is exactly the form adopted in Ref. [24].

Finally, we mention that, exactly in the same way as in Ref. [24], we introduce an isospin correction in the matrix elements of $W^{\downarrow}$ in the $T_{-}$channel. In fact, it must be noted that in this case the coupling with the intermediate states $|N\rangle$ can mix states with different isospins. These intermediate states, which contain a phonon plus a proton particle and a neutron hole, do not have pure isospin. They can be written as

$$
|N\rangle=c_{-1}\left|N ; T_{0}-1, T_{0}-1\right\rangle+c_{0}\left|N ; T_{0}, T_{0}-1\right\rangle+c_{+1}\left|N ; T_{0}+1, T_{0}-1\right\rangle,
$$

where $T_{0}=(N-Z) / 2$, and the coefficients $c_{i}$ are simply Clebsch-Gordan coefficients,

$$
\begin{aligned}
c_{-1} & =\left\langle 1-1 T_{0} T_{0} \mid T_{0}-1, T_{0}-1\right\rangle=\left(2 T_{0}-1\right)^{1 / 2} /\left(2 T_{0}+1\right)^{1 / 2}, \\
c_{0} & =\left\langle 1-1 T_{0} T_{0} \mid T_{0}, T_{0}-1\right\rangle=-\left(T_{0}+1\right)^{-1 / 2}, \\
c_{+1} & =\left\langle 1-1 T_{0} T_{0} \mid T_{0}+1, T_{0}-1\right\rangle=1 /\left[\left(T_{0}+1\right)^{1 / 2}\left(2 T_{0}+1\right)^{1 / 2}\right] .
\end{aligned}
$$

(this writing is justified by the fact that our phonons, as mentioned below, are to a good approximation isoscalar phonons). The $\mathrm{GT}^{-}$resonance has isospin quantum numbers $\left|T, T_{z}\right\rangle=\left|T_{0}-1, T_{0}-1\right\rangle$, and its coupling with states of different isospin should be forbidden by the nuclear Hamiltonian. We impose that it is strictly forbidden (this amounts to neglecting Coulomb effects in the residual interaction) and, therefore, we project out the isospin component with the same value of the $\mathrm{GT}^{-}$resonance in the intermediate states, i.e., the component $T=T_{0}-1$. Correspondingly, in the $W^{\downarrow}$ matrix elements, an isospin correction factor $\left|c_{-1}\right|^{2}=\left(2 T_{0}-1\right) /\left(2 T_{0}+1\right)$ is added.

\section{NUMERICAL DETAILS}

In the present work, the HF equations are solved in coordinate space on a radial mesh whose size is $0.1 \mathrm{fm}$, within a box of $21 \mathrm{fm}$. The p-h configuration space used for the GT RPA calculation includes all hole states and particle states (discretized in the mentioned box) up to an upper cutoff $E_{\text {cut }}=100 \mathrm{MeV}$. The results are fully converged in this way

(for example, the Ikeda sum rule of ${ }^{60} \mathrm{Ni}$ with the interaction SGII [27] reaches 11.998 at the RPA level with this cutoff).

To build the model space of the p-h pairs plus phonon doorway states, needed for $W^{\downarrow}$, the energies and reduced transition probabilities of the most collective phonon modes with 
TABLE I: Properties of low-lying phonons in ${ }^{60} \mathrm{Ni}$. The theoretical results are calculated with the Skyrme interactions SGII [27], SLy5 [28], and $\mathrm{SkM}^{*}$ [29]. The experimental data are taken from Ref. [30].

\begin{tabular}{|c|c|c|c|c|c|}
\hline \multirow[t]{4}{*}{ State } & \multicolumn{3}{|c|}{ Theory } & \multirow{2}{*}{\multicolumn{2}{|c|}{$\begin{array}{c}\text { Experiment } \\
\text { Energy } B(E L, 0 \rightarrow L)\end{array}$}} \\
\hline & Energy & $B(E L, 0 \rightarrow L$ & & & \\
\hline & {$[\mathrm{MeV}]$} & {$\left[\mathrm{e}^{2} \mathrm{fm}^{2 L}\right]$} & & {$[\mathrm{MeV}]$} & {$\left[\mathrm{e}^{2} \mathrm{fm}^{2 L}\right]$} \\
\hline & SGII SLy5 SkM* & SLy5 & $\mathrm{SkM}^{*}$ & & \\
\hline $2_{1}^{+}$ & 1.8201 .1572 .212 & $3.611 \times 10^{2} 3.562 \times 10^{2}$ & $2.457 \times 10^{2}$ & 1.333 & $8.780 \times 10^{2}$ \\
\hline $3_{1}^{-}$ & 4.8975 .7274 .901 & $1.576 \times 10^{4} 1.643 \times 10^{4}$ & $1.150 \times 10^{4}$ & 4.040 & \\
\hline $4_{1}^{+}$ & $1.902 \quad 2.343 \quad 3.469$ & $1.007 \times 10^{5} 7.257 \times 10^{4}$ & $6.365 \times 10^{4}$ & 2.506 & \\
\hline
\end{tabular}

spin and parity $2^{+}, 3^{-}, 4^{+}$are calculated with the same energy cutoff. With this choice in, e.g., ${ }^{60} \mathrm{Ni}$, the energy-weighted sum rules (EWSRs) satisfy the double commutator values by about $98 \%$. Non-charge-exchange RPA is implemented in exactly the same way as chargeexchange RPA, that is, with the same numerical input.

To minimize violations of the Pauli principle, and be consistent with the very idea of particle-vibration coupling, only phonons which absorb a fraction of the total isoscalar or isovector strength larger than 5\% (and with energy less than $20 \mathrm{MeV}$ ) are included in the model space $Q_{2}$. The properties of the low-lying $2_{1}^{+}, 3_{1}^{-}$, and $4_{1}^{+}$states in ${ }^{60} \mathrm{Ni}$ calculated with three different Skyrme interactions, namely SGII, SLy5, and SkM*, are shown in Table I.

We finally point out that we have checked, in the present calculation of Gamow-Teller resonances, that the Ikeda sum rule is still satisfied at the level of RPA plus particle-vibration coupling. For instance, in the case of ${ }^{60} \mathrm{Ni}$ calculated with the Skyrme interactions SGII, SLy5, and $\mathrm{SkM}^{*}, m_{0}\left(\mathrm{GT}^{-}\right)-m_{0}\left(\mathrm{GT}^{+}\right)=11.87,11.89$, and 11.83, respectively, when the strength is integrated up to $45 \mathrm{MeVfor}{ }^{56} \mathrm{Ni}$, the sum rule value is -0.10 for the interaction SGII, -0.07 for SLy5, and -0.11 for SkM* when the strength is integrated up to $45 \mathrm{MeV}$; and for ${ }^{208} \mathrm{~Pb}$ it is 129.29 for the interaction SGII when the strength is integrated up to $70 \mathrm{MeV}$. 
TABLE II: Correspondence between numbers and interactions in Fig. 2, Fig. 3, and Fig. 4,

\begin{tabular}{cccccccccccccc}
\hline \hline Number & 1 & 2 & 3 & 4 & 5 & 6 & 7 & 8 & 9 & 10 & 11 & 12 & 13 \\
\hline Interaction SLy5 SLy4 & SkP LNS SkI3 & SkM* & Sk255 SIII BSk17 & SGII SkO' SkI4 SkO \\
Reference & {$[28]$} & {$[28]$} & {$[31]$} & {$[32]$} & {$[33]$} & {$[29]$} & {$[34]$} & {$[35]$} & {$[36]$} & {$[27]$} & {$[37]$} & {$[33]$} & {$[37]$} \\
\hline \hline
\end{tabular}

\section{SENSITIVITY OF THE RPA RESULTS TO THE CHOICE OF THE SKYRME SET}

We have first tried to understand the sensitivity of the GT energies to the choice of the Skyrme interaction used for RPA, and the underlying reasons.

The main components of the GT response are the transitions between spin-orbit partners. In a nucleus such as ${ }^{60} \mathrm{Ni}$, which is our benchmark in this study, the $\mathrm{GT}^{-}\left(\mathrm{GT}^{+}\right)$response is expected to be dominated by the single transition $\nu f_{7 / 2} \rightarrow \pi f_{5 / 2}\left(\pi f_{7 / 2} \rightarrow \nu f_{5 / 2}\right)$. In such a case, we can approximately write the energy of the GTR as

$$
E_{\mathrm{RPA}}=E_{\text {unper }}+E_{\mathrm{res}} \approx \Delta E_{l s}+V \text {. }
$$

The first term $E_{\text {unper }}$ is the unperturbed p-h energy, and can be associated with the spinorbit splitting $\Delta E_{l s}$ whereas the second term $E_{\text {res }}$ is the energy shift induced by the residual interaction and can be written in terms of its matrix element $V$. The matrix elements of the residual interaction in the spin-isospin channel are as a rule repulsive, so the two terms of Eq. (26) have the same sign.

In the case of a Skyrme calculation, the first term is controlled by the spin-orbit parameter $W_{0}$ (we leave aside the nonstandard Skyrme sets that have two parameters in the spin-orbit part of the energy functional, and those which include the so-called $\mathbf{J}^{2}$ terms). The second term depends instead on the strength of the residual interaction in the spin-isospin channel. In general one imagines that it is mainly associated with the well-known Landau parameter $g_{0}^{\prime}$, but we should not forget that $g_{1}^{\prime}$ plays a role as well (these are the only two Landau parameters that do not vanish for a zero-range interaction). In Ref. [5], by studying the strength of the $\mathrm{GT}^{-}$resonance in several nuclei including $\mathrm{Sn}$ isotopes and ${ }^{208} \mathrm{~Pb}$, we have concluded that this strength is sensitive to $g_{0}^{\prime}$ and $g_{1}^{\prime}$, and that values around 0.45 and 0.5 are preferable for these parameters.

In the present study, we focus first on the energy position of both the $\mathrm{GT}^{-}$and $\mathrm{GT}^{+}$ 

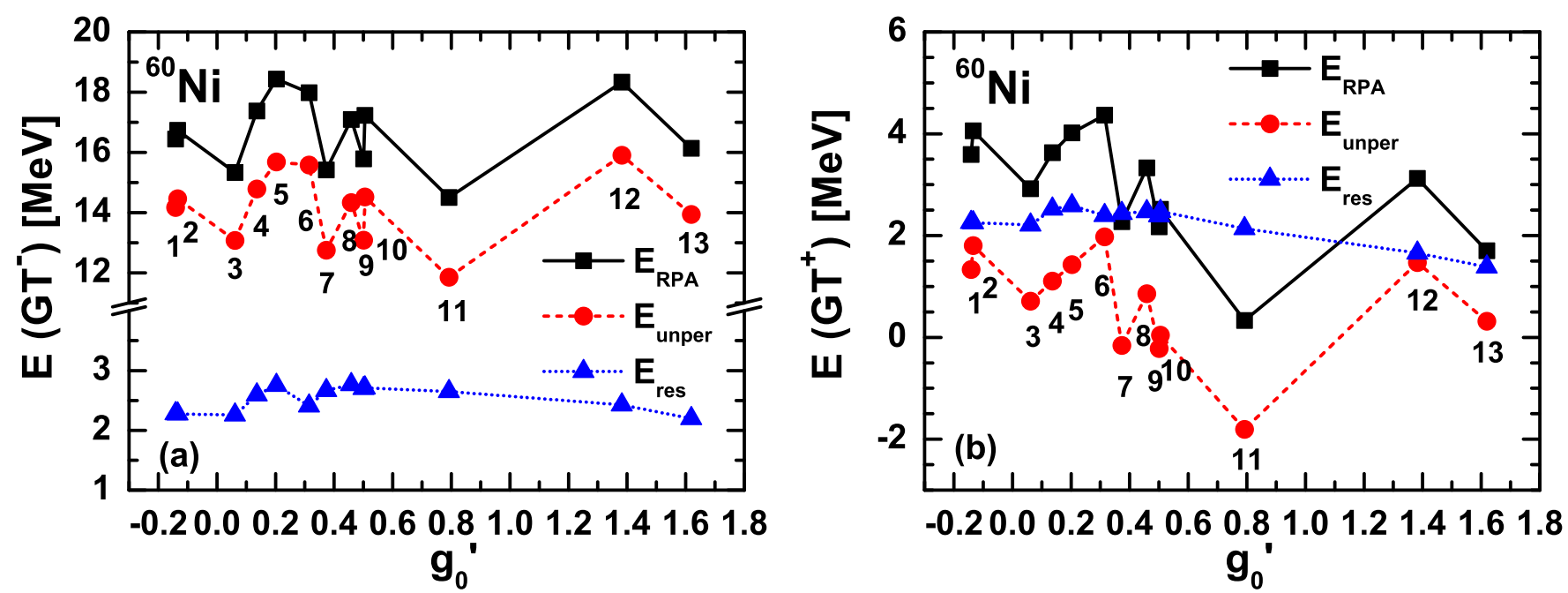

FIG. 2: (Color online) The Gamow-Teller energy calculated in the RPA ( $\left.E_{\mathrm{RPA}}\right)$, the unperturbed energy of the main p-h components $\left(E_{\text {unper }}\right.$, see the text), and the associated shift $E_{\text {res }} \equiv E_{\mathrm{RPA}}-$ $E_{\text {unper }}$ are displayed as functions of the Landau parameter $g_{0}^{\prime}$ for nucleus ${ }^{60} \mathrm{Ni}$. The $\mathrm{GT}^{-}$and $\mathrm{GT}^{+}$ cases are in panel (a) and panel (b), respectively, and the correspondence between numbers and Skyrme sets can be found in Table II.

peaks. In Fig. 2, the following quantities are displayed, as a function of $g_{0}^{\prime}$ : the main peak of the Gamow-Teller resonance calculated in RPA, the unperturbed Gamow-Teller energy of the single p-h configuration $\left(\pi f_{5 / 2}\right)\left(\nu f_{7 / 2}\right)^{-1}$ for $\mathrm{GT}^{-}$or $\left(\nu f_{5 / 2}\right)\left(\pi f_{7 / 2}\right)^{-1}$ for $\mathrm{GT}^{+}$, and the shift $E_{\mathrm{res}} \equiv E_{\mathrm{RPA}}-E_{\text {unper }}$. Surprisingly, the energy shift is flat as a function of $g_{0}^{\prime}$ : we have found that this is due to a strong cancellation between the contributions associated with $g_{0}^{\prime}$ and $g_{1}^{\prime}$. The staggering of the $\mathrm{GT}^{-}$main peak is associated with the staggering in the unperturbed energy (which is obviously uncorrelated with the Landau parameters), and it follows it quite closely. The same can be said of the $\mathrm{GT}^{+}$peak; in this case the absolute value of the unperturbed energy is, however, smaller than in the previous case and smaller than the typical matrix element of the residual force.

In Fig. 3 the RPA and unperturbed energies are again displayed as a function of the spin-orbit strength parameter $W_{0}$. Only a subset of all the studied interaction has been chosen for this figure. The dashed and dash-dotted lines are linear fits of the points that are displayed only as guides to the eye. A quantitative correlation between the parameter $W_{0}$ and the GT energy can be neither inferred from the numerical results nor expected, since the unperturbed energy is associated with a neutron-proton transition. However, it is clear 

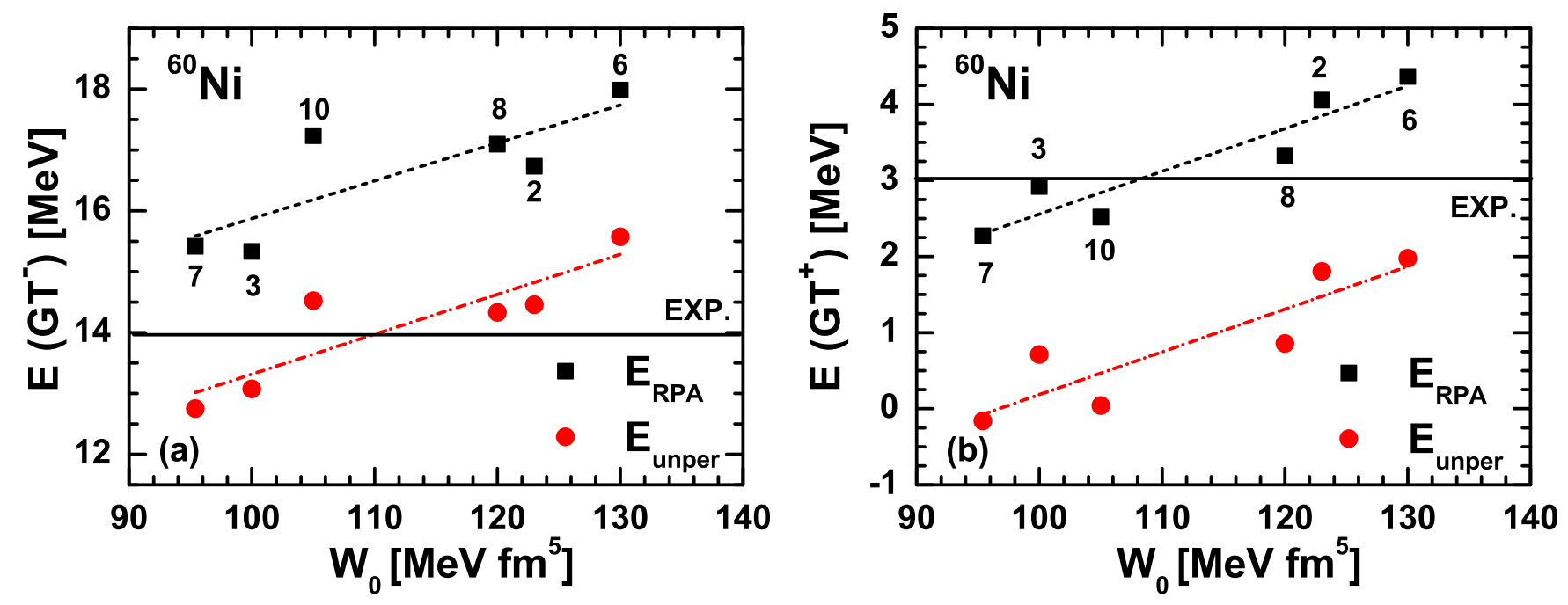

FIG. 3: (Color online) The same energies $E_{\mathrm{RPA}}$ and $E_{\text {unper }}$ shown in Fig. 2 are here displayed as functions of $W_{0}$. The lines are linear fits that are, however, shown only for illustrative purposes. See the text for a detailed discussion. The experimental values for the energies (expressed with respect to the parent nucleus as in the theory) are indicated by means of black solid lines and are taken from Ref. [38] for the $\mathrm{GT}^{-}$case and from Ref. [39] for the $\mathrm{GT}^{+}$case.

that there is a qualitative tendency of both unperturbed and RPA energies to grow as a function of $W_{0}$, and that these energies are more sensitive to the single-particle spectrum than to other parameters such as $g_{0}^{\prime}$ or $g_{1}^{\prime}$.

In the same figure we also display, by means of horizontal lines, the experimental values of the energies taken from Refs. [38, 39]. Since the particle-vibration coupling produces, as a rule, a downward shift of the resonance energies (see the next section), we are inclined to prefer, for further considerations, the sets marked as 2, 6, 8, and (at least looking at the $\mathrm{GT}^{-}$case) 10. Set 8 is SIII, which is a force that does not have very satisfactory properties as a whole (for instance, the associated value of the nuclear incompressibility is too large). Set 2 is SLy4, but SLy5 provides quite similar results. Sets 6 and 10 are, respectively, SkM* and SGII. We will discuss in the next section some results obtained by using SkM*, SLy5, and SGII. SkM* and SGII have reasonable values for the Landau parameters [5].

In Ref. [5] it has been found, however, that the parameters $g_{0}^{\prime}$ and $g_{1}^{\prime}$ are not irrelevant for the GT properties, in keeping with the fact that they are correlated with the $\mathrm{GT}^{-}$strength in ${ }^{208} \mathrm{~Pb}$ as well as in $\mathrm{Sn}$ isotopes. We have checked here what happens for the $\mathrm{GT}^{-}$energy in ${ }^{208} \mathrm{~Pb}$, and our results are shown in Fig. 4, Although the single-particle properties still 


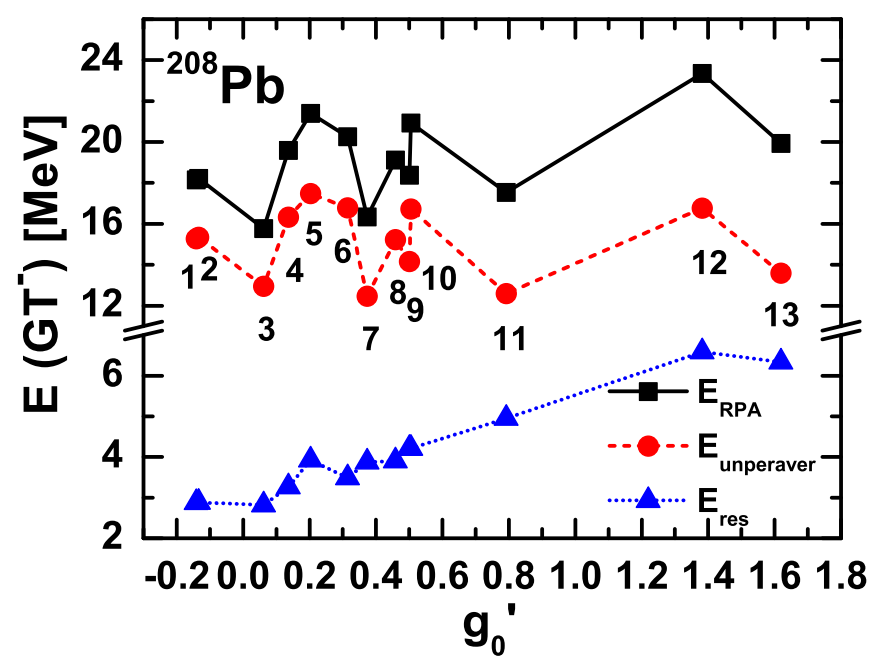

FIG. 4: (Color online) The same quantities already displayed in Fig. 2 are now displayed in the case of ${ }^{208} \mathrm{~Pb}$. Here, the unperturbed energy $E_{\text {unperaver }}$ is the weighted average of the two main configurations $\nu i_{13 / 2} \rightarrow \pi i_{11 / 2}$ and $\nu h_{11 / 2} \rightarrow \pi h_{9 / 2}$.

play a relevant role - as can be seen from the staggering that is similar to that in the figure for ${ }^{60} \mathrm{Ni}$ - here there is a clear tendency of the shift associated with the residual interaction: it is not flat, rather it grows to some extent with $g_{0}^{\prime}$. We have found that in fact there is less cancellation between the terms associated with $g_{0}^{\prime}$ and $g_{1}^{\prime}$. Moreover, in the schematic model, the RPA energy is known to scale as

$$
E_{\mathrm{RPA}} \approx E_{\text {unperaver }}+n V
$$

where $n$ is the number of p-h configurations, $E_{\text {unperaver }}$ is the weighted average of the unperturbed energies of the $\mathrm{n}$ configurations contributing to the RPA state. This number is not 1 here, as it was in the case of ${ }^{60} \mathrm{Ni}$. The role of the residual interaction must be larger here, accordingly.

In conclusion, the GT energy is, within the Skyrme framework, very sensitive to the single-particle spectrum. This is especially true in nuclei of the $f p$ shell (although we have discussed only ${ }^{60} \mathrm{Ni}$ in some detail, we have reached similar conclusions from the analysis of ${ }^{56,58} \mathrm{Ni}$ and ${ }^{54,56} \mathrm{Fe}$ ). There is some kind of tendency for both the $\mathrm{GT}^{-}$and $\mathrm{GT}^{+}$energies to grow as a function of the spin-orbit parameter $W_{0}$. Our analysis has shown that a few Skyrme sets may be suitable to be employed for PVC calculations with the expectation that the experimental energy can be well reproduced. 

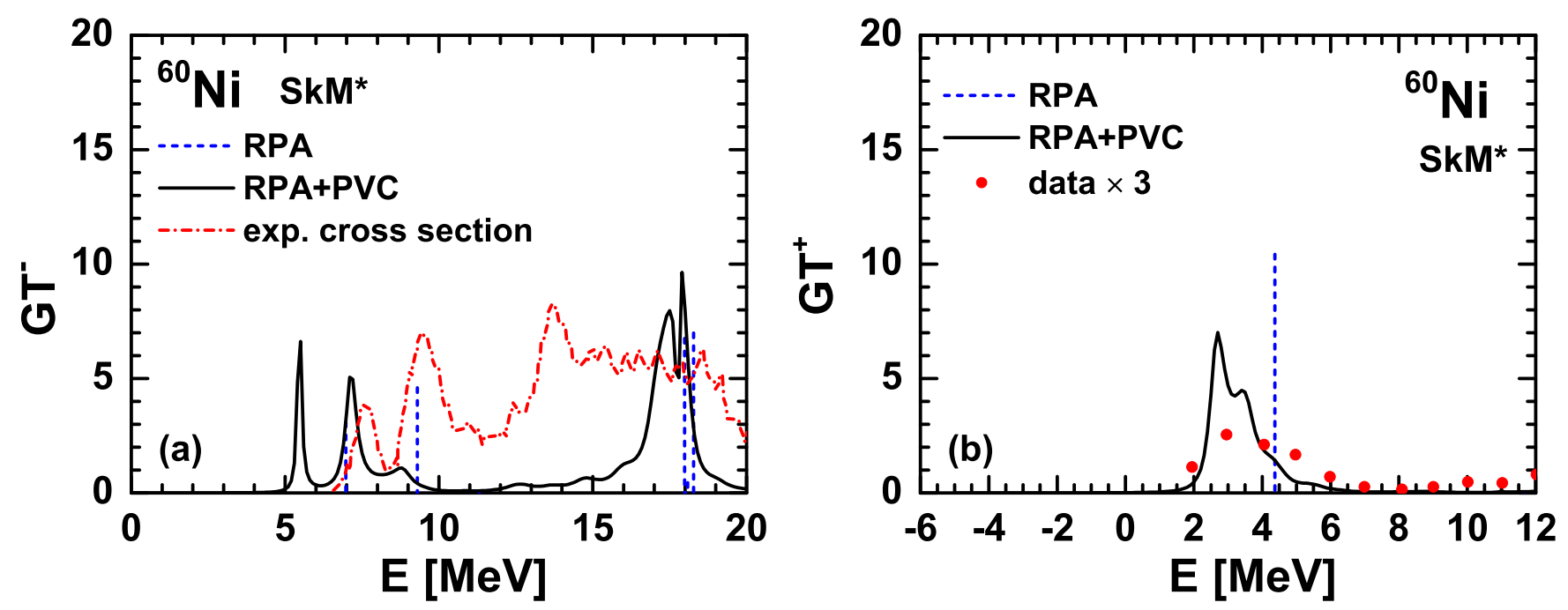

FIG. 5: (Color online) Gamow-Teller strength distributions calculated with the Skyrme interaction $\mathrm{SkM}^{*}$ for the nucleus ${ }^{60} \mathrm{Ni}$. The blue dashed discrete lines denote the RPA strength (with dimensionless units), and the black solid lines represent the distribution (with units of $\mathrm{MeV}^{-1}$ ) calculated by the RPA+PVC model. The experimental data for the case of $\mathrm{GT}^{-}$[38] [with units of $\mathrm{mb} /(\mathrm{sr} \mathrm{MeV})]$ and $\mathrm{GT}^{+}$[39] (with units of $\mathrm{MeV}^{-1}$ ), displayed by either a red dash-dotted line or red points, are discussed in the text.

\section{RPA WITH PARTICLE VIBRATION COUPLING}

In the present section we discuss the results of the calculations that include the coupling with vibrations. We use the interactions SkM*, SLy5, and SGII, following the discussion in the previous section. The goal is to see if, and to what extent, the coupling with vibrations decreases the energies (in particular the $\mathrm{GT}^{+}$threshold energy) and changes the distribution of the strength. Of course we are also interested in seeing if RPA plus PVC can reproduce the total resonance width. In our previous work for ${ }^{208} \mathrm{~Pb}$ [24], we have seen that the coupling with vibrations can account for most of the spreading width of the GTR.

\section{A. Energies and widths of the GT states in ${ }^{60} \mathrm{Ni}$}

The Gamow-Teller strength distributions as a function of the energy (with respect to the parent nuclei) are displayed in Figs. 5, 6, and 7in the case of the Skyrme interactions SkM*, Sly5, and SGII, respectively, for the nucleus ${ }^{60} \mathrm{Ni}$. The blue dashed discrete lines denote the 
RPA strength, and the black solid lines represent the strength distribution calculated by the RPA +PVC model. From all figures, it is clear that the inclusion of phonon coupling leads to two significant effects for both the $\mathrm{GT}^{-}$and the $\mathrm{GT}^{+}$case: there is a downward shift of the excitation energy, and the development of a spreading width. Especially in the high-energy region, a single $\mathrm{RPA} \mathrm{GT}^{-}$peak spreads into a wide resonance covering an energy region of the order of $5 \mathrm{MeV}$ (or more).

The development of a realistic spreading width allows, in principle, a full comparison of the theoretical results with the experimental data. The spreading width is caused by the coupling with the phonons, through the imaginary part of $W^{\downarrow}$. In the case of $\mathrm{GT}^{-}$, a detailed strength distribution is not found in Ref. [38], so only the measured zero-degree cross section is displayed in Figs. 5, 6 and 7 by means of a dash-dotted red line (cf. the left panels). All three interactions produce two peaks in the low-energy part, and one main high-lying GTR. These qualitative features are in agreement with the experimental findings. However, the energies of the low-lying peaks are underestimated, and the GTR is not as broad as in the experiment. One can say we can account for a relevant fraction of the measured total width, yet the high-lying experimental cross section looks extremely flat.

In the case of the $\mathrm{GT}^{+}$the experimental data from Ref. [39] are indicated by points. The total width of the $\mathrm{GT}^{+}$distribution is about $3 \mathrm{MeV}$. The theoretical result obtained with the force $\mathrm{SkM}^{*}$ agrees very well with experiment, as far as both the peak energy and the width are concerned. SLy5 also reproduces well the width, whereas in SGII one finds two peaks but still a fragmentation of the strength over a reasonable energy interval. It must be noted that the experimental data are multiplied by a factor of 3 in the right panels of Figs. 5, 6 and 7. We discuss this issue in the next subsection.

\section{B. The quenching problem}

As a result of the phonon coupling, we can say we redistribute the Gamow-Teller strength in a region that is several $\mathrm{MeV}$ wide. However, this does not produce a real quenching. In the case of ${ }^{60} \mathrm{Ni}$ with the force SGII, up to the energy of $20 \mathrm{MeV}(10 \mathrm{MeV})$, the integrated $\mathrm{GT}^{-}\left(\mathrm{GT}^{+}\right)$strength calculated by RPA+PVC is 19.4 (8.1). For comparison, the RPA value is 20.8 (9.3). Consequently, the strength is quenched by $7 \%$ (13\%) compared with RPA but it is larger than the experimental data. In experimental results, one finds a total 
strength equal to $7.2 \pm 1.8$ in the case of the $\mathrm{GT}^{-}$[38] (up to $21 \mathrm{MeV}$ in the parent nucleus), and $3.11 \pm 0.08$ in the case of the $\mathrm{GT}^{+}$[39] (up to $10 \mathrm{MeV}$ in the parent nucleus). In the calculation of RPA+PVC with interaction SGII, only $15 \%$ of the sum rule shifts to energies above $20 \mathrm{MeV}$.

SkM* and SLy5 give similar (i.e., small) quenching effects as SGII. In the case of SLy5, one finds an integrated strength of 23.4 up to $20 \mathrm{MeV}$ for the $\mathrm{GT}^{-}$case after coupling with phonons (instead of 24.8 given by RPA). For the $\mathrm{GT}^{+}$case, the integrated strength up to 10 $\mathrm{MeV}$ in RPA+PVC is 12.1 (instead of 13.0 from RPA). 11\% of the total sum rule is shifted to the energy region $20-45 \mathrm{MeV}$. In the case of $\mathrm{SkM}^{*}$, the integrated strength for $\mathrm{GT}^{-}$ $\left(\mathrm{GT}^{+}\right)$from RPA+PVC is $20.3(9.5)$ up to $20 \mathrm{MeV}(10 \mathrm{MeV})$, instead of 21.9 (10.4) from RPA. $15 \%$ percent of the sum rule is shifted to the energy region $20-45 \mathrm{MeV}$.

There are probably two missing effects that can explain the lack of quenching in our calculations (let alone the small, or negligible, coupling with the $\Delta$ isobar). In Ref. [11] a large shift of strength to high energies is found by coupling with uncorrelated $2 \mathrm{p}-2 \mathrm{~h}$ doorway states. These extend up to higher energy, as compared with the $1 \mathrm{p}-1 \mathrm{~h}$ plus phonon doorway states. Consequently, although less important for the spreading width, they can account for the shift of strength above $20 \mathrm{MeV}$. In that work, the tensor force has been found to play a role as well. Even in the limited $1 \mathrm{p}-1 \mathrm{~h}$ space, the tensor force has been found to be capable of shifting strength at high energy, although in smaller amounts [7].

\section{Microscopic origin of the downward shift induced by phonon coupling}

In general, it can be expected that the coupling with phonons leads to a downward shift of the resonance peaks, and that the low-lying vibrations are the most effective. We analyze here in detail the effects in the case of the $\mathrm{GT}^{+}$peak of Fig. 7.

The total shift in the peak energy for the $\mathrm{GT}^{+}$channel is $-2.1 \mathrm{MeV}$, and the $2^{+}, 3^{-}, 4^{+}$ phonons give partial contributions equal to $-1.3,-0.8$, and $-0.1 \mathrm{MeV}$, respectively. For every multipole, the lowest phonon, which is the most collective one, plays the most important role, giving $85 \%(-1.1 \mathrm{MeV})$ and $75 \%(-0.6 \mathrm{MeV})$ of the shift for the $2^{+}$and $3^{-}$ case, respectively. The diagonal matrix element $W_{p h, p h}^{\downarrow}$, when the $p h$ configuration is the dominant component of the $\mathrm{GT}^{+}$peak $\left[\nu 1 f_{5 / 2}\left(\pi 1 f_{7 / 2}\right)^{-1}\right]$, is identified as the dominant term responsible for the energy shift. In the case of coupling with the first $2^{+}$phonon, the first 
[Fig. 1 (1)] and second [Fig. 1 (2)] diagrams of $W_{p h, p h}^{\downarrow}$ give similar negative contributions to the energy shift both with values of about $-1.3 \mathrm{MeV}$, while the third [Fig. 1 (3)] and fourth [Fig. 1 (4)] diagrams cancel them by about 70\%. These numbers are calculated at the value of $\omega$ at which the GT peak lies if we couple the RPA state with the $2_{1}^{+}$state only, namely $\omega=1.4 \mathrm{MeV}$. In the first and second diagrams of Fig. 1, the $2^{+}$phonons can only couple to the intermediate states with negative parity. It turns out that the particle states $\nu 1 f_{5 / 2}$ and $\nu 2 p_{1 / 2}$ are the most important intermediate states for the first graph, and provide contributions accounting for $62 \%(-0.8 \mathrm{MeV})$ and $23 \%(-0.3 \mathrm{MeV})$ of the total value of the first diagram $(-1.3 \mathrm{MeV})$, while the intermediate hole state $\pi 1 f_{7 / 2}$ is the most important one for the second graph, giving almost all the contribution to the total value of the second diagram $(-1.3 \mathrm{MeV})$. In the case of coupling with the first $3^{-}$phonons, the first and second diagrams still give similar negative contributions to the energy shift (both about $-0.3 \mathrm{MeV}$, these values being calculated at the value of $\omega$ at which the GT peak lies if we couple the RPA state with the $3_{1}^{-}$state only, namely $\omega=1.9 \mathrm{MeV}$ ); however, the

third and fourth diagrams are zero in the case of $W_{p h, p h}^{\downarrow}$ when $p h$ is $\nu 1 f_{5 / 2}\left(\pi 1 f_{7 / 2}\right)^{-1}$, due to parity conservation. In this matrix element, the $3^{-}$phonons only couple with intermediate states with positive parity. The states $\nu 1 g_{9 / 2}, \nu 1 g_{7 / 2}, \nu 2 d_{5 / 2}$ are the most important particle states and the associated contributions to the first diagram of Fig. 1 are $-0.05,-0.05$, and $-0.03 \mathrm{MeV}$ (the total value of the first diagram is $-0.3 \mathrm{MeV}$ ). Similarly, in the second diagram the important hole states are $\pi 2 s_{1 / 2}, \pi 1 d_{5 / 2}, \pi 1 d_{3 / 2}$, and the contributions are -0.13 , -0.08 , and $-0.05 \mathrm{MeV}$ (the total value of the second diagram is $-0.3 \mathrm{MeV}$ ).

\section{Results for the nucleus ${ }^{56} \mathrm{Ni}$}

Since the $\mathrm{GT}^{-}$resonance in the nucleus ${ }^{56} \mathrm{Ni}$ was recently measured [23], we also present results for this nucleus, calculated with the three interactions SkM*, SLy5, and SGII (cf. Figs. 8], and 10, respectively). Similarly to ${ }^{60} \mathrm{Ni}$, the coupling with phonons shifts the peaks downward and produces a spreading width, yet brings a quite limited quenching effect. The energy shift caused by the phonon coupling is about $1-2 \mathrm{MeV}$ for all three interactions. The energy calculated by means of SGII and SLy5 is somewhat lower than the experimental data, while the energy from $\mathrm{SkM}^{*}$ agrees very well with them. In this latter case $\left(\mathrm{SkM}^{*}\right)$ the strength spreads out in the same energy region as the experimental strength distribution. In 

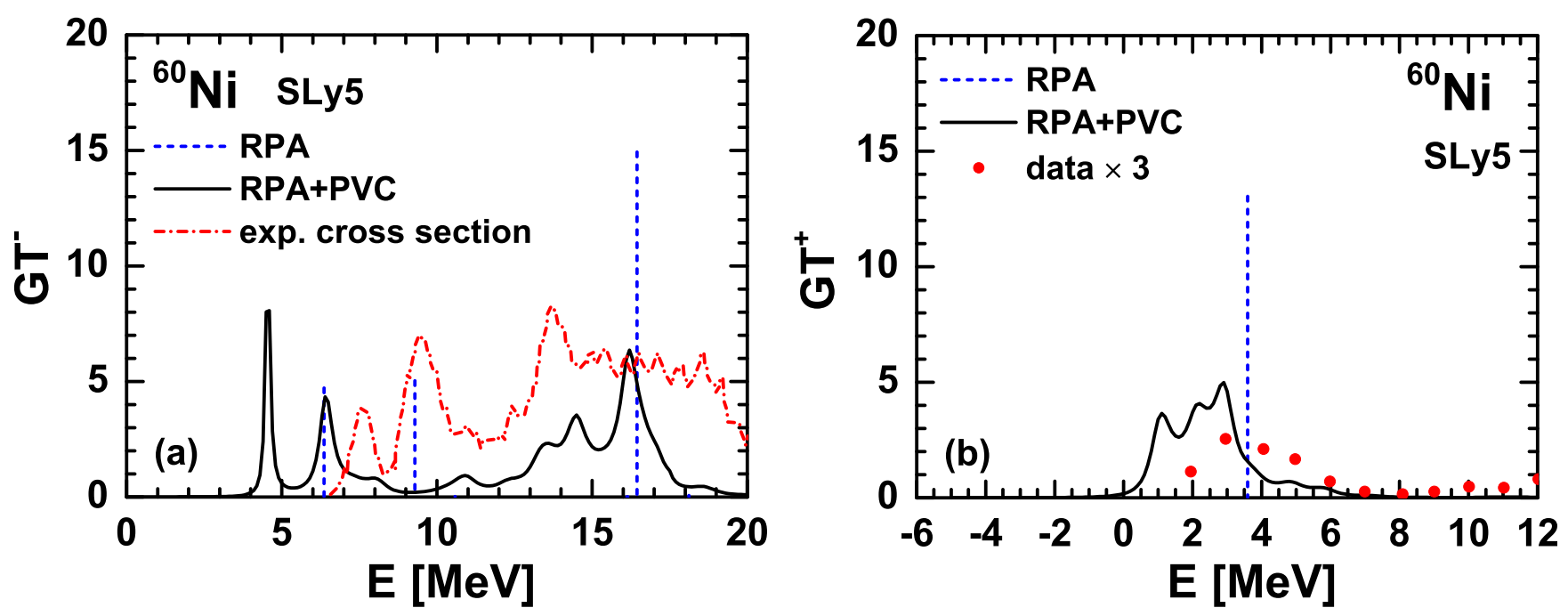

FIG. 6: (Color online) The same as Fig. 5 in the case of the Skyrme interaction SLy5.
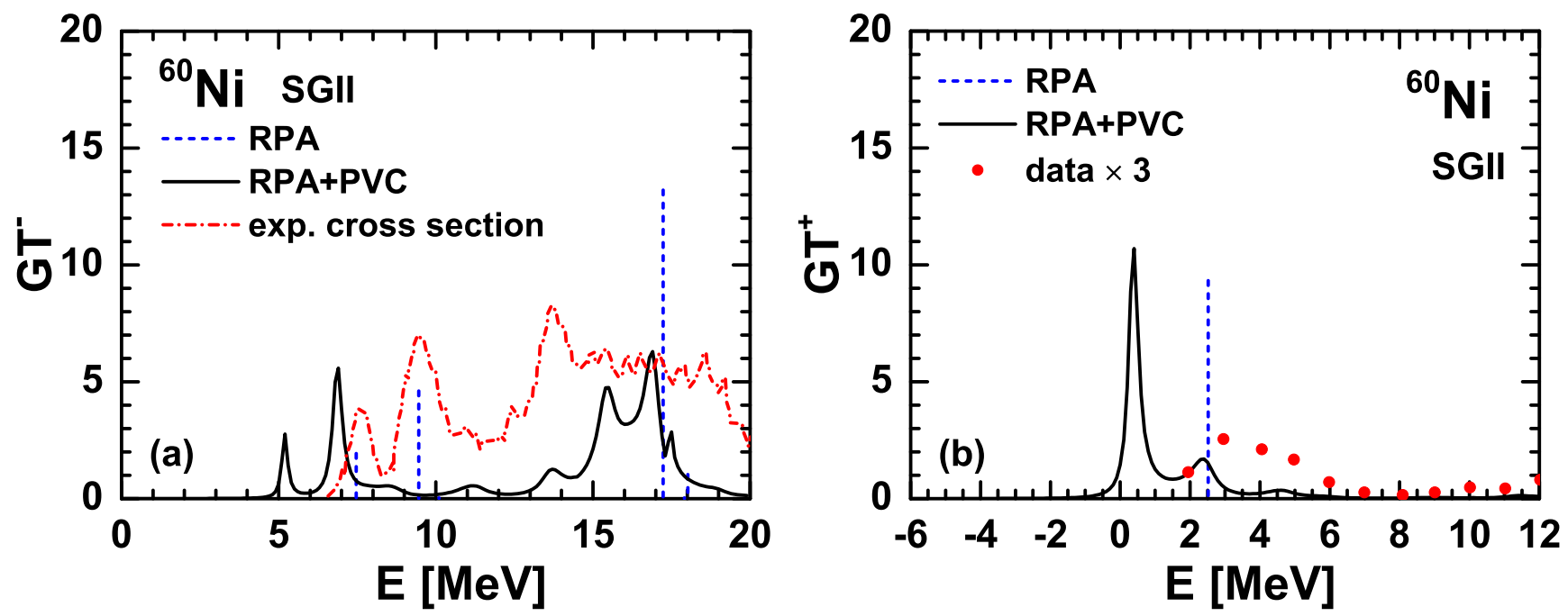

FIG. 7: (Color online) The same as Fig. [5 in the case of the Skyrme interaction SGII.

experimental results, there is a double-peak structure and each peak has a width of about $2 \mathrm{MeV}$. In our calculations, we also obtain a similar structure in the strength distribution for all three interactions. For SGII and SLy5, the first peak has a width of about $1 \mathrm{MeV}$ and the second peak has a width of about $2 \mathrm{MeV}$. For SkM*, the width is about $1.5 \mathrm{MeV}$ for the first peak and $1 \mathrm{MeV}$ for the second one. The big difference between our results and the experimental data is the absolute value of the strength. As in ${ }^{60} \mathrm{Ni}$, the coupling with phonons does not induce a significant quenching (see the above discussion). In the energy region $10-24 \mathrm{MeV}$, after the coupling with phonons, the integrated strength becomes 


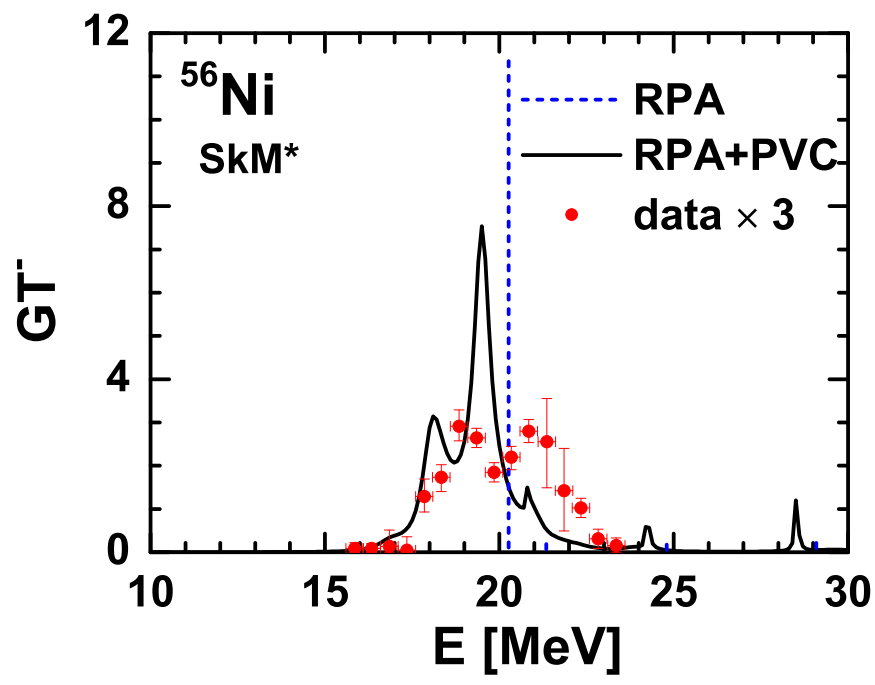

FIG. 8: (Color online) Gamow-Teller strength distributions calculated with the Skyrme interaction $\mathrm{SkM}^{*}$ for the nucleus ${ }^{56} \mathrm{Ni}$. The blue dashed discrete line denotes the RPA strength (with dimensionless units), and the black solid line represents the distribution (with units of $\mathrm{MeV}^{-1}$ ) calculated by the RPA+PVC model. The experimental data (with units of $\mathrm{MeV}^{-1}$ ) from Ref. [23] are indicated by red points.

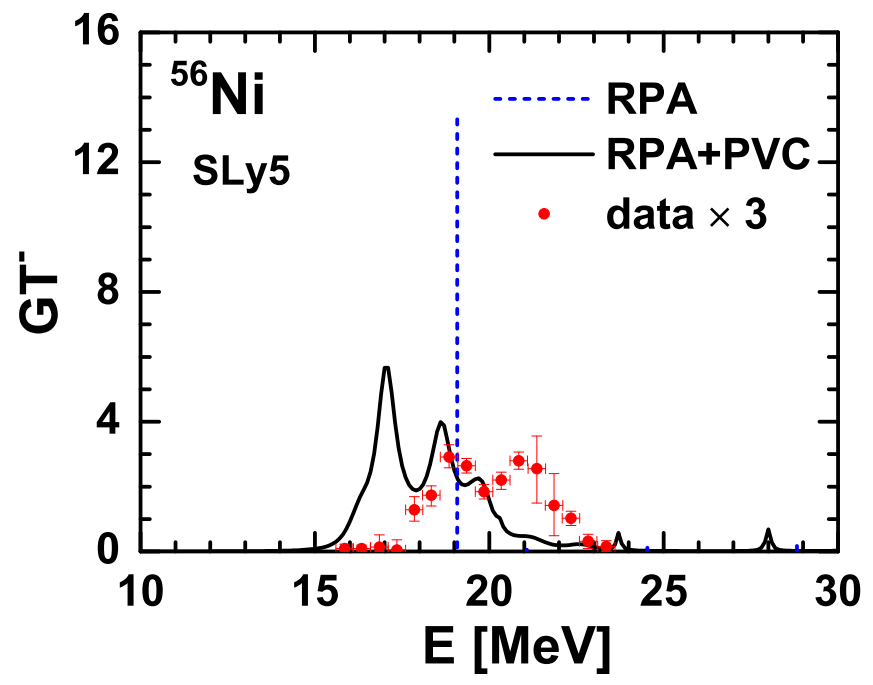

FIG. 9: (Color online) The same as Fig. 8 in the case of the Skyrme interaction SLy5.

10.3, 12.8, and 10.8 (instead of 11.0,13.4, and 11.5 calculated in RPA), for the interactions SGII, SLy5, and SkM*, respectively. The same value is $3.5 \pm 0.3 \pm 1.0$ from experiment [23]. 


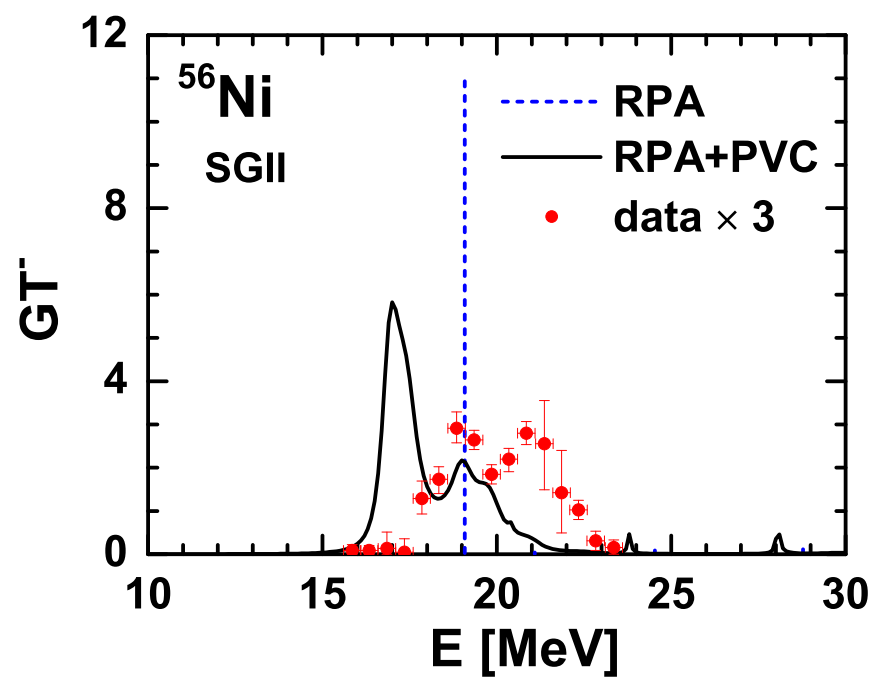

FIG. 10: (Color online) The same as Fig. 8 in the case of the Skyrme interaction SGII.

\section{CONCLUSION}

In the present work, we have analyzed the features of the Gamow-Teller strength distributions in nuclei of the $f p$ shell. These nuclei are of interest for astrophysical applications, but few have been also objects of experimental measurements. Our purpose is to improve existing models so that they can reproduce the experimental findings, whenever available, and become reliable for calculations of the electron capture rates needed for astrophysical simulations; our ambition is to avoid resorting to free, ad hoc parameters.

We have first performed RPA calculations with many different Skyrme parameter sets. We have seen that the energy of the $\mathrm{GT}^{-}$and $\mathrm{GT}^{+}$peak is quite sensitive to the singleparticle properties and in particular, to a large extent, to the spin-orbit strength parameter. The energy shift caused by residual interaction is less relevant to the Landau parameter $g_{0}^{\prime}$ for these medium-mass nuclei than in the case of, e.g., ${ }^{208} \mathrm{~Pb}$, the reason being the smaller number of p-h configurations.

Coupling with phonons is relevant to producing a more realistic strength distribution, characterized by a spreading width. The interaction SkM* does reproduce the peak position and the spreading width, in the cases of the $\mathrm{GT}^{+}$resonance in ${ }^{60} \mathrm{Ni}$ and the $\mathrm{GT}^{-}$ resonance in ${ }^{56} \mathrm{Ni}$, after the phonon coupling is taken into account. This is important for the astrophysical applications since the $\mathrm{GT}^{+}$energy is associated with the threshold energy for electron capture. Other interactions display nonetheless, in the same nuclei, realistic 
strength fragmentation after the phonon coupling is taken into account.

We omit a full description of the GT strength quenching. While in shell-model calculations this is often accounted for by means of a free parameter, we refrain from making this choice here. Our model probably can be improved by including coupling with doorway states extending to higher energy, and by taking care of the tensor force as well.

\section{ACKNOWLEDGMENTS}

This work was partly supported by the Major State 973 Program No. 2007CB815000, by the National Natural Science Foundation of China under Grants No. 10975008 and No. 11175002, and by the Research Fund for the Doctoral Program of Higher Education under Grant No. 20110001110087. The support of the Italian Research Project "Many-body theory of nuclear systems and implications on the physics of neutron stars" (PRIN 2008) is also acknowledged, as it has allowed the visit of one of the authors (Y.N.) in Milano. 
[1] F. Osterfeld, Rev. Mod. Phys. 64, 491 (1992).

[2] M. Bender, J. Dobaczewski, J. Engel, and W. Nazarewicz, Phys. Rev. C 65, 054322 (2002).

[3] N. Paar, T. Nikšić, D. Vretenar, and P. Ring, Phys. Rev. C 69, 054303 (2004).

[4] N. Paar, D. Vretenar, E. Khan, and G. Colò, Rep. Prog. Phys. 70, 691 (2007).

[5] S. Fracasso and G. Colò, Phys. Rev. C 76, 044307 (2007).

[6] H. Liang, N. Van Giai, and J. Meng, Phys. Rev. Lett. 101, 122502 (2008).

[7] C. Bai, H. Sagawa, H. Zhang, X. Zhang, G. Colò, and F. Xu, Phys. Lett. B 675, 28 (2009).

[8] K. Ikeda, S. Fujii, and J. I. Fujita, Phys. Lett. 3, 271 (1963).

[9] C. Gaarde, Nucl. Phys. A 396, 127 (1983).

[10] T. Wakasa, H. Sakai, H. Okamura, H. Otsu, S. Fujita, S. Ishida, N. Sakamoto, T. Uesaka, Y. Satou, M. B. Greenfield, et al., Phys. Rev. C 55, 2909 (1997).

[11] G. F. Bertsch and I. Hamamoto, Phys. Rev. C 26, 1323 (1982).

[12] S. Drozdz, V. Klemt, J. Speth, and J. Wambach, Phys. Lett. B 166, 18 (1986).

[13] I. S. Towner and J. C. Hardy, Rep. Prog. Phys. 73, 046301 (2010).

[14] H. Liang, N. Van Giai, and J. Meng, Phys. Rev. C 79, 064316 (2009).

[15] K. Langanke and G. Martínez-Pinedo, Rev. Mod. Phys. 75, 819 (2003).

[16] H. A. Bethe, G. E. Brown, J. Applegate, and J. M. Lattimer, Nucl. Phys. A 324, 487 (1979).

[17] H. A. Bethe, Rev. Mod. Phys. 62, 801 (1990).

[18] H.-T. Janka, K. Langanke, A. Marek, G. Martínez-Pinedo, and B. Müller, Phys. Rep. 442, 38 (2007).

[19] D. Dean, K. Langanke, L. Chatterjee, P. Radha, and M. Strayer, Phys. Rev. C 58, 536 (1998).

[20] K. Langanke, E. Kolbe, and D. Dean, Phys. Rev. C 63, 032801 (2001).

[21] N. Paar, G. Colò, E. Khan, and D. Vretenar, Phys. Rev. C 80, 055801 (2009).

[22] Y. F. Niu, N. Paar, D. Vretenar, and J. Meng, Phys. Rev. C 83, 045807 (2011).

[23] M. Sasano, G. Perdikakis, R. G. T. Zegers, S. M. Austin, D. Bazin, B. A. Brown, C. Caesar, A. L. Cole, J. M. Deaven, N. Ferrante, et al., Phys. Rev. Lett. 107, 202501 (2011).

[24] G. Colò, N. Van Giai, P. F. Bortignon, and R. A. Broglia, Phys. Rev. C 50, 1496 (1994).

[25] P. Ring and P. Schuck, The Nuclear Many-Body Problem (Springer-Varlag Berlin Heidelberg, 1980). 
[26] G. Colò, H. Sagawa, and P. F. Bortignon, Phys. Rev. C 82, 064307 (2010).

[27] N. Van Giai and H. Sagawa, Phys. Lett. B 106, 379 (1981).

[28] E. Chabanat, P. Bonche, P. Haensel, J. Meyer, and R. Schaeffer, Nucl. Phys. A 635, 231 (1998).

[29] J. Bartel, P. Quentin, M. Brack, C. Guet, and H.-B. Håkansson, Nucl. Phys. A 386, 79 (1982).

[30] http://www.nndc.bnl.gov.

[31] J. Dobaczewski, H. Flocard, and J. Treiner, Nucl. Phys. A 422, 103 (1984).

[32] L. G. Cao, U. Lombardo, C. W. Shen, and N. V. Giai, Phys. Rev. C 73, 014313 (2006).

[33] P.-G. Reinhard and H. Flocard, Nucl. Phys. A 584, 467 (1995).

[34] B. K. Agrawal, S. Shlomo, and V. Kim Au, Phys. Rev. C 68, 031304 (2003).

[35] M. Beiner, H. Flocard, N. V. Giai, and P. Quentin, Nucl. Phys. A 238, 29 (1975).

[36] S. Goriely, N. Chamel, and J. M. Pearson, Phys. Rev. Lett. 102, 152503 (2009).

[37] P.-G. Reinhard, D. J. Dean, W. Nazarewicz, J. Dobaczewski, J. A. Maruhn, and M. R. Strayer, Phys. Rev. C 60, 014316 (1999).

[38] J. Rapaport, T. Taddeucci, T. Welch, C. Gaarde, J. Larsen, D. Horen, E. Sugarbaker, P. Koncz, C. Foster, C. Goodman, et al., Nucl. Phys. A 410, 371 (1983).

[39] A. L. Williams, W. P. Alford, E. Brash, B. A. Brown, S. Burzynski, H. T. Fortune, O. Häusser, R. Helmer, R. Henderson, P. P. Hui, et al., Phys. Rev. C 51, 1144 (1995). 